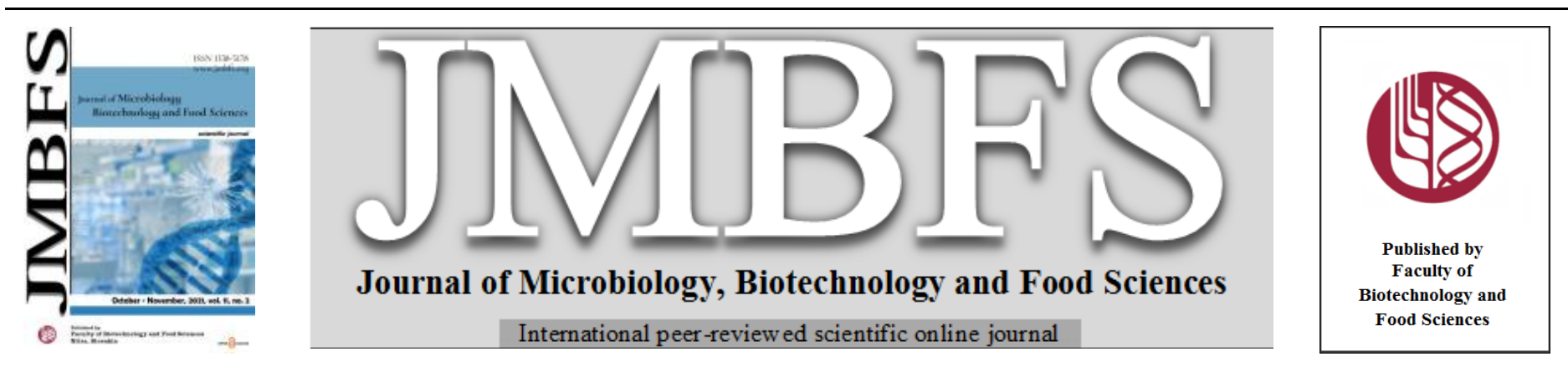

\title{
ANTIHYPERLIPIDEMIC EFFECT OF TEPHROSIA VILLOSA IN ACUTE AND CHRONIC HYPERLIPIDEMIA RAT MODELS
}

\author{
Vimal John Samuel ${ }^{1}$, Vedigounder Murugan ${ }^{* 2}$, Agasa Ramu Mahesh ${ }^{2}$ \\ Address(es): \\ ${ }^{1}$ College of Pharmaceutical Sciences, Dayananda Sagar University, Department of Pharmacology, Kumaraswamy Layout, 560078, Bengaluru, Karnataka, India, +91 \\ 8892022234. \\ ${ }^{2}$ College of Pharmaceutical Sciences, Dayananda Sagar University, Department of Pharmaceutical Chemistry, Kumaraswamy Layout, 560078, Bengaluru, Karnataka, \\ India.
}

*Corresponding author: vimal-sps@dsu.edu.in

https://doi.org/10.15414/jmbfs.4222

\section{ARTICLE INFO}

Received 16. 1. 2021

Revised 10. 4. 2021

Accepted 13. 4. 2021

Published 1. 10. 2021

Regular article

open ${ }_{\text {ACCESS }}$

\begin{abstract}
Atherosclerosis plays a pivotal role in various cardiovascular disorders. Most of the antiatherogenic currently available drugs are associated with many side effects than plant derived formulations. Tephrosia villosa (Fabaceae) plant is being used traditionally, in the treatment of hyperlipidemia, diabetes, jaundice and used as antioxidant and antimicrobial. The objective of the current experiment is to evaluate the antihyperlipidemic potency of chloroform (CETV) and ethanolic extract of Tephrosia villosa (EETV) in acute (Triton WR1339 induced) and chronic (cholesterol diet-induced) hyperlipidemia models. Simvastatin, standard drug used to compare the effect of the both extracts at $125 \mathrm{mg} / \mathrm{kg}, 250 \mathrm{mg} / \mathrm{kg}$ and $500 \mathrm{mg} / \mathrm{kg}$ b.w). The serum lipid parameters were analysed using enzymatic kits. The preliminary phytochemical screening of extracts of Tephrosia villosa showed the presence of phenols, flavonoids, saponins, coumarins, glycosides and alkaloids. In acute model, the pretreatment with both extracts for 7 days showed significant reduction $(\mathrm{p}<0.001)$ in serum triglycerides (TG), total cholesterol (TC), very low-density lipoprotein cholesterol (VLDL-C) and low-density lipoprotein cholesterol $($ LDL-C) levels, and subsequent rise $(\mathrm{p}<0.01)$ in high-density lipoprotein cholesterol (HDL-C) levels after 24h tritonisation in hyperlipidemic rats. Similarly, in chronic hyperlipidemia model, all the lipid (TG, TC, LDL-C \& VLDL-C) levels were reduced significantly $(\mathrm{p}<0.001)$ with successive rise in HDL-C level after 30 days of study with 15 days of extract treatment. The current study indicates the antihyperlipidemic activity of Tephrosia villosa is probably due to the phenolic constituents in extracts, which may inhibit HMG CoA reductase or activate lipoprotein lipase and lecithin acyl transferase (LCAT). The results would provide a larger insight in design and development of newer therapeutics for a wide arena of lifestyle disorders.
\end{abstract}

Keywords: Tephrosia villosa, Triton WR-1339, Cholesterol, Simvastatin, LDL-C, HDL-C, Atherogenic index

\section{INTRODUCTION}

Hyperlipidemia is defined as alterations in lipid metabolism characterised by collateral increase in plasma lipids such as phospholipids, total cholesterol, triglycerides, LDL-C and VLDL-C and HDL-C (Nelson et al., 2013; Kreisberg et al., 2005; Sikarwar et al., 2014). It is generally classified as primary (Familial) which could be due to genetics, and secondary caused by underlying disorder. Hyperlipidemia provides the well-established associations in causing cardiovascular diseases (CVD), as a result of lipid deposition, calcification and migration of muscle cells to intima layer in arteries (E Souza et al., 2017). It is also believed to increase the death rate by $50 \%$ (Karr et al., 2017). Thus, diagnosing and management of hyperlipidemia is necessary.

Statins, fibric acid derivatives, selective cholesterol absorption inhibitors and PSCK 9 (Proprotein Convertase Subtilsin-Kexin type 9) inhibitor are few lipid lowering drugs available in the market for the treatment (Zodda et al., 2018). Although there are many pharmaceutical products available for therapeutic management of hyperlipidemia, many people prefer the nutraceuticals due to their lesser side effects, less expensive and its natural (Santini et al., 2017). This favour the discovery of new lead molecule in treating hyperlipidemia of various aetiology through different regulating mechanism (Gupta et al., 2018). Hence, the current researchers were focusing on new lead components from natural sources.

The Tephrosia genus is belongs to the family Leguminosae, a large group of plant consists of more than 400 species worldwide where most of them grown as shrub and weeds. They primarily distributed in Africa, Australia, America and Asia. Moreover, in India 24 species were identified in which Tephrosia villosa is one among them. This genus is rich in prenylated flavonoids and traditionally used as antihyperlipidemic, antidiabetic, antimicrobial and antioxidant (Samuel et al., 2019; Mani et al., 2017; Krishnasamy et al., 2019). Several previous studies showed the effect on lipid parameters are due to the presence of polyphenols like flavonoids, triterpenoid, sterol, retinoid and triterpene in various plants (Hmidani $\boldsymbol{e t}$ al.,
2020). According to Namdeo, most of the marketed products are directly or indirectly derived from the plant derived products called Green medicine, is believed to be safe and trustworthy. Hence the curiosity about the plant derived medicine has been special interest for researcher and for many pharmaceutical companies who are concentrating on substantial research on plants products for their medicinal value (Kumar et al., 2016).

\section{MATERIALS AND METHODS}

\section{Collection of plant material}

Tephrosia villosa, a perennial under shrub found largely in Kolar district of Karnataka, India, was collected as whole plant. The collected plant material was authenticated by Dr. V. Rama Rao, Research Officer (Scientist-2) Botany, Regional Ayurveda Research Institute for Metabolic Disorders, Bengaluru. The herbarium specimen of the collected plant was conserved for future reference in the department.

\section{Extraction}

The entire plant of Tephrosia villosa was sliced down into small pieces, shade dried for 45 days and grounded to get moderately coarse powder, which is sieved under mesh. About $1000 \mathrm{~g}$ of coarse powder of plant was subjected for extraction process using with chloroform and ethanol at $50^{\circ}-60^{\circ} \mathrm{C}$ for $72 \mathrm{~h}$ by using soxhlet apparatus. The chloroform and ethanolic extracts are obtained successively and subsequently concentrated to obtain residue by using vacuum distillation. The extracts obtained was subjected for phytochemical screening.

\section{Preliminary phytochemical studies}

Chloroform (CETV) and ethanolic (EETV) extract of Tephrosia villosa are subjected for preliminary phytochemical investigation for qualitative 
identification to discover phytochemical constituents present in the extract (Gokhale et al., 2016).

\section{Experimental Animals}

The male Wistar albino rats (180 - $220 \mathrm{~g})$ were procured from Sri Raghavendra Enterprises, Bangalore. As per standard laboratory conditions, rats were housed in polyacrylic cages for $12 \mathrm{~h}$ light/dark cycle at the temperature of $26 \pm 2{ }^{\circ} \mathrm{C}$ and relative humidity $44-56 \%$, and standard food and water ad libitum throughout the study. About 7 days of acclimatization of animals to laboratory conditions was subjected before the test. Institutional Animal Ethics Committee (IAEC) approval (DSU/Ph.D/IAEC/19/2018-19) was obtained prior to the experiment and studies were performed as per CPCSEA guidelines.

\section{Acute oral toxicity studies}

Organization for economic cooperation and development (OECD) guidelines, class method 423 was used to perform acute oral toxicity study for CETV and EETV. The study was carried out to determine the $\mathrm{LD}_{50}$ of the extracts in female albino Wistar rats weighing between 180 and 220 g. About $300 \mathrm{mg} / \mathrm{kg} \mathrm{b.w.p.o}$ was taken as initial dose and increased up to $5000 \mathrm{mg} / \mathrm{kg} \mathrm{b.w.} \mathrm{p.o.} \mathrm{to} \mathrm{observe}$ signs of toxicity and mortality (Guideline, O. E. C. D., 2002).

\section{Induction of Hyperlipidemia}

Triton WR-1339 (200 mg/kg b.w), used intraperitoneally to induce acute hyperlipidemia on the $7^{\text {th }}$ day of the study (Ibrahim et al., 2016). On the other hand, oral administration of cholesterol diet (cholesterol $400 \mathrm{mg} / \mathrm{kg}+$ coconut oil as a vehicle, p.o) for 30 days was followed to induce chronic hyperlipidemia (Vijaya et al., 2009).

\section{Pharmacological activities}

\section{Acute hyperlipidemia model}

To observe the short-term effect of CETV and EETV on Triton-induced hyperlipidemia, rats were allotted into nine groups comprising six rats in each group $(n=6)$. Group I served as normal control (NC), which received 1\% CMC (vehicle) throughout the study. Group II received Triton WR-1339 (200 mg/kg b.w i.p) which served as hyperlipidemic control (HC). Group III (STD) served as standard, received Simvastatin at $10 \mathrm{mg} / \mathrm{kg}$ b.w p.o dose. (Hmidani et al., 2020). Group IV to VI received CETV $125 \mathrm{mg} / \mathrm{kg}, 250 \mathrm{mg} / \mathrm{kg}$ and $500 \mathrm{mg} / \mathrm{kg} \mathrm{b.w} \mathrm{p.o}$ respectively. Similarly, group VII to IX received EETV $125 \mathrm{mg} / \mathrm{kg}, 250 \mathrm{mg} / \mathrm{kg}$ and $500 \mathrm{mg} / \mathrm{kg}$ b.w p.o respectively. All rats were treated with respective drug treatments from day 0 to day 7 . On $7^{\text {th }}$ day animals of group II to IX were treated with Triton WR-1339 (200 mg/kg; i.p), immediately after drug administration. After 6 and $24 \mathrm{~h}$ tritonisation, blood samples were collected from all the rats by retro-orbital puncture which was further processed into serum and used for estimation of lipid parameters (Dubey et al., 2005).

\section{Chronic hyperlipidemia model}

Induction of hyperlipidemia in rats was achieved using cholesterol rich diet to analyse the antihyperlipidemic effect of CETV and EETV. The rats were grouped similar to Triton-induced hyperlipidemic model where all animals were fed with cholesterol diet $(400 \mathrm{mg} / \mathrm{kg}$, p.o) except normal control (NC) group (Group I), received normal diet throughout 30 days of the study. Group II served as hyperlipidemic control (HC), which received only cholesterol diet. Group III (STD) rats received Simvastatin $(10 \mathrm{mg} / \mathrm{kg}$ b.w p.o $)$ whereas Group IV to VI received CETV $125 \mathrm{mg} / \mathrm{kg}, 250 \mathrm{mg} / \mathrm{kg}$ and $500 \mathrm{mg} / \mathrm{kg}$ b.w p.o respectively. Similarly, group VII to IX received EETV $125 \mathrm{mg} / \mathrm{kg}, 250 \mathrm{mg} / \mathrm{kg}$ and $500 \mathrm{mg} / \mathrm{kg}$ b.w p.o respectively. Animals were gavaged with respective drug treatments from day 16 to day 30 . Blood samples were collected on $0^{\text {th }}, 15^{\text {th }}$ and $30^{\text {th }}$ from the retro-orbital vein of the rats (Vijaya et al., 2009; Sampathkumar et al., 2011).

\section{Estimation of serum lipid profile}

The overnight fasted animals were anesthetized with pentobarbitone $(50 \mathrm{mg} / \mathrm{kg}$ b.w i.p) on last day of the study. The blood samples were collected in the eppendorfs tube, kept in upright position for approximately 10-15 $\mathrm{min}$ to facilitate blood clotting. The sample was centrifuged at $3000 \mathrm{rpm}$ for $15 \mathrm{~min}$. The separated serum was used for the estimations of TG, TC, HDL-C and LDL-C levels by using enzymatic kits (Erba diagnostics, Germany). The VLDL-C level and Atherogenic index (AI) were determined by equations given below:

$\mathrm{VLDL}=\mathrm{TG} / 5, \mathrm{AI}=\mathrm{TC} / \mathrm{HDL}-\mathrm{C}($ Khazaal, 2013)

\section{Statistical analysis}

All the values were specified in Mean \pm SEM (Standard Error of Mean) GraphPad instat (GraphPad Software Inc.) was used to find the statistical differences $(\mathrm{p}<0.05)$ between the groups using one-way ANOVA and followed by Tukey multiple comparison test.

\section{RESULTS}

\section{Preliminary phytochemical studies of Tephrosia villosa extracts}

The phytoconstituents present in the CETV and EETV was obtained after phytochemical evaluation. On phytochemical screening the presence of phenols, flavonoids, saponins, glycosides, alkaloids, fixed oils and fats were revealed.

Table 1. Phytochemical investigation of extracts of Tephrosia villosa

\begin{tabular}{llcc}
\hline S.No & Chemical constituents & $\begin{array}{c}\text { Chloroform } \\
(\text { CETV })\end{array}$ & Ethanol (EETV) \\
\hline 1. & Alkaloids & + & + \\
\hline 2. & Carbohydrates & - & - \\
\hline 3. & Glycosides & + & + \\
\hline 4. & Saponins & + & + \\
\hline 5. & Phytosterols & - & - \\
\hline 6. & Fats and oils & + & + \\
\hline 7. & Resins & - & - \\
\hline 8. & Flavonoids & + & + \\
\hline 9. & Proteins & - & - \\
\hline 10. & Steroids & - & - \\
\hline 11. & Phenolic compounds & + & + \\
\hline 12. & Tannins & - & - \\
\hline
\end{tabular}

+ Present, - Absent, CETV: Chloroform extract of T.villosa, EETV: Ethanolic extract of T.villosa

\section{Acute oral toxicity studies of Tephrosia villosa extracts}

During the period of observation (14 days) no mortality was exhibited up to 5000 $\mathrm{mg} / \mathrm{kg} \mathrm{b.w}$. dose and also there was no gross distinct effect on general motor activity, feeding behaviour, muscular weakness, faecal output etc seen by both CETV and EETV extracts. Thus, the dose for further pharmacological study was selected as $125 \mathrm{mg} / \mathrm{kg}, 250 \mathrm{mg} / \mathrm{kg}$ and $500 \mathrm{mg} / \mathrm{kg}$ b.w p.o for both the extracts.

\section{Effect of Tephrosia villosa extracts on lipid profile Acute hyperlipidemia model}

As anticipated, after $6 \mathrm{~h}$ and $24 \mathrm{~h}$ of administration (i.p) of Triton in hyperlipidemic control (HC) group resulted in significant $(\mathrm{p}<0.001)$ increase in TC, TG, VLDL-C and LDL-C levels, and marked decrease in HDL-C level, related to normal control (NC) group. In case of pretreatment with CETV and EETV at 3 different doses $(125 \mathrm{mg} / \mathrm{kg}, 250 \mathrm{mg} / \mathrm{kg}$ and $500 \mathrm{mg} / \mathrm{kg}$ b.w) exhibited dose-dependent effect on serum lipid parameters. Both CETV and EETV had showed highly significant ( $\mathrm{p}<0.001$ ) suppression of LDL-C, VLDL-C, TC and TG levels and marked increase $(\mathrm{p}<0.01)$ in HDL-C level after $24 \mathrm{~h}$ tritonisation at high dose $(500 \mathrm{mg} / \mathrm{kg} \mathrm{b.w})$, whereas moderated changes in serum lipid parameters at $250 \mathrm{mg} / \mathrm{kg} \mathrm{b} . \mathrm{w}$ dose and no significant effect at $125 \mathrm{mg} / \mathrm{kg} \mathrm{b} . \mathrm{w}$ dose. But the results obtained after $6 \mathrm{~h}$ tritonisation for both extracts showed moderate changes in lipid parameters only at $500 \mathrm{mg} / \mathrm{kg} \mathrm{b.w}$ dose, and no significant changes with other two doses. On the other hand, these findings were compared with the standard drug Simvastatin which showed a remarkable reduction $(p<0.001)$ in serum lipid parameter expect HDL-C in group III (STD). All the above said results were shown in Table 2 and Figure 1. 
Table 2 Effect of chloroform and ethanolic extract of $T$. villosa on lipid profile in hyperlipidemic rats of acute model

\begin{tabular}{|c|c|c|c|c|c|c|c|c|}
\hline \multirow{2}{*}{ Groups } & \multicolumn{2}{|c|}{ TG mg/dL } & \multicolumn{2}{|c|}{ TC mg/dL } & \multicolumn{2}{|c|}{ LDL-C mg/dL } & \multicolumn{2}{|c|}{ HDL-C mg/dL } \\
\hline & $6 \mathrm{~h}$ & $24 \mathrm{~h}$ & $6 \mathrm{~h}$ & $24 \mathrm{~h}$ & $6 \mathrm{~h}$ & $24 \mathrm{~h}$ & $6 \mathrm{~h}$ & $24 \mathrm{~h}$ \\
\hline NC & $81.16 \pm 4.82$ & $82.23 \pm 3.09$ & $60.32 \pm 2.21$ & $61.18 \pm 2.92$ & $20.15 \pm 1.47$ & $21.49 \pm 1.95$ & $34.48 \pm 2.26$ & $36.21 \pm 3.86$ \\
\hline HC & $188.36 \pm 5.17^{* * *(a)}$ & $308.16 \pm 6.06^{* * *(a)}$ & $170.96 \pm 4.16^{* * *(a)}$ & $281.64 \pm 4.37^{* * *(a)}$ & $94.06 \pm 3.49^{* * *(a)}$ & $165.15 \pm 4.32^{* * *(a)}$ & $24.16 \pm 1.22^{* *(a)}$ & $22.16 \pm 1.26^{* * *(a)}$ \\
\hline STD & $151.19 \pm 4.25^{* * *(b)}$ & $201.47 \pm 3.27^{* * *(b)}$ & $142.96 \pm 3.46^{* * *(b)}$ & $198.78 \pm 5.86^{* * *(b)}$ & $59.12 \pm 3.41^{* * *(b)}$ & $127.03 \pm 5.02^{* * *(b)}$ & $33.18 \pm 2.33^{*(b)}$ & $38.48 \pm 1.31^{* * * *(b)}$ \\
\hline $\begin{array}{l}\text { CETV } \\
(125 \mathrm{mg} / \mathrm{kg})\end{array}$ & $174.19 \pm 4.17^{\mathrm{ns}}$ & $280.62 \pm 5.16^{*(\mathrm{~b})}$ & $154.25 \pm 5.61^{\mathrm{ns}}$ & $263.29 \pm 5.14^{\mathrm{ns}}$ & $89.41 \pm 4.39^{\mathrm{ns}}$ & $154.61 \pm 4.18^{\mathrm{ns}}$ & $26.85 \pm 2.17^{\mathrm{ns}}$ & $21.98 \pm 2.36^{\mathrm{ns}}$ \\
\hline $\begin{array}{l}\text { CETV } \\
(250 \mathrm{mg} / \mathrm{kg})\end{array}$ & $178.41 \pm 2.47^{\mathrm{ns}}$ & $271.62 \pm 5.42^{* *(b)}$ & $166.17 \pm 4.17^{\mathrm{ns}}$ & $234.15 \pm 6.93^{* * *(b)}$ & $96.64 \pm 4.03^{\mathrm{ns}}$ & $142.46 \pm 3.67^{*(b)}$ & $25.05 \pm 1.09^{\mathrm{ns}}$ & $29.19 \pm 0.84^{*(b)}$ \\
\hline $\begin{array}{l}\text { CETV } \\
(500 \text { mg/kg) }\end{array}$ & $156.74 \pm 2.41^{* * *(b)}$ & $216.81 \pm 1.96^{* * *(b)}$ & $151.25 \pm 3.28^{* *(b)}$ & $209.54 \pm 3.06^{* * *(b)}$ & $80.26 \pm 2.03^{*(b)}$ & $108.43 \pm 4.12^{* * *(b)}$ & $29.14 \pm 1.39^{\mathrm{ns}}$ & $32.12 \pm 1.21^{* *(b)}$ \\
\hline $\begin{array}{l}\text { EETV } \\
(125 \mathrm{mg} / \mathrm{kg})\end{array}$ & $180.11 \pm 5.35^{\mathrm{ns}}$ & $289.22 \pm 5.43^{\mathrm{ns}}$ & $158.57 \pm 6.15^{\mathrm{ns}}$ & $267.91 \pm 4.48^{\mathrm{ns}}$ & $92.18 \pm 4.19^{\mathrm{ns}}$ & $160.28 \pm 4.87^{\mathrm{ns}}$ & $30.11 \pm 2.03^{\mathrm{ns}}$ & $24.38 \pm 2.06^{\mathrm{ns}}$ \\
\hline $\begin{array}{l}\text { EETV } \\
(250 \mathrm{mg} / \mathrm{kg})\end{array}$ & $172.83 \pm 3.24^{*(b)}$ & $279.32 \pm 4.57^{* *(b)}$ & $169.26 \pm 5.08^{\mathrm{ns}}$ & $251.27 \pm 4.11^{* * *(b)}$ & $93.58 \pm 3.08^{\mathrm{ns}}$ & $154.46 \pm 2.67^{\mathrm{ns}}$ & $24.05 \pm 1.09^{\mathrm{ns}}$ & $28.24 \pm 1.41^{*(\mathrm{~b})}$ \\
\hline $\begin{array}{l}\text { EETV } \\
(500 \mathrm{mg} / \mathrm{kg})\end{array}$ & $169.74 \pm 2.97^{* *(b)}$ & $238.97 \pm 4.43^{* * *(b)}$ & $160.33 \pm 4.18^{\mathrm{ns}}$ & $221.16 \pm 6.46^{* * *(b)}$ & $95.62 \pm 3.75^{\mathrm{ns}}$ & $120.54 \pm 4.56^{* * *(b)}$ & $28.65 \pm 1.03^{\mathrm{ns}}$ & $30.57 \pm 2.11^{* *(b)}$ \\
\hline
\end{tabular}

Values are expressed as Mean \pm SEM;

${ }^{a}$ Values were against normal control (NC).

${ }^{\mathrm{b}}$ Values were against hyperlipidemic control (HC)

Values are statistically significant at $* * * \mathrm{p}<0.001,{ }^{* *} \mathrm{p}<0.01, * \mathrm{p}<0.05$ and ${ }^{\mathrm{ns}}$ nonsignificant.

NC: Normal control, HC: Hyperlipidemic control, STD: Standard control (Simvastatin), CETV: Chloroform extract of T.villosa, EETV: Ethanolic extract of T.villosa, TC: Total cholesterol, TG: Triglycerides, HDL-C: High density lipoprotein cholesterol, LDL-C: Low density lipoprotein cholesterol

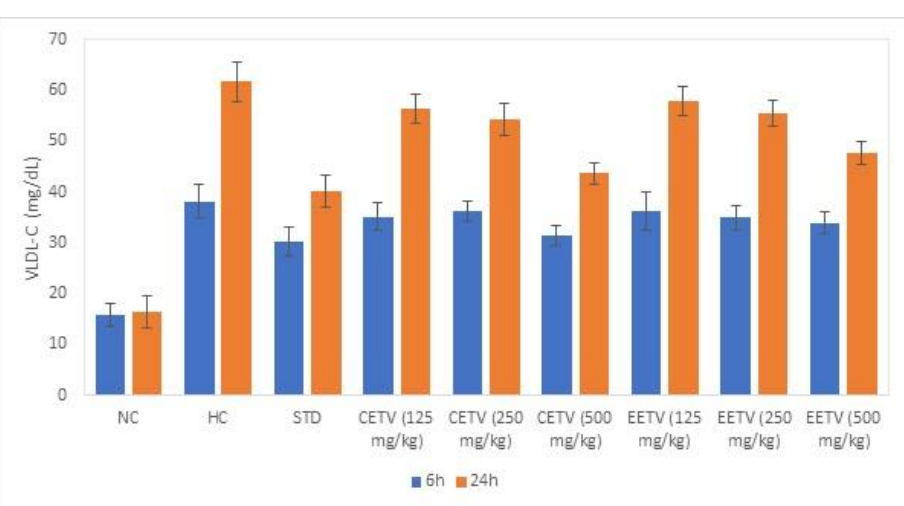

Figure 1 Effect of chloroform and ethanolic extract of T. villosa on VLDL-C level in hyperlipidemic rats of acute model.

Furthermore, atherogenic index (AI) was evaluated and showed that there is an increase in AI of hyperlipidemic control $(\mathrm{HC})$ group in contrast to normal control (NC) group. But there was a dose dependent reduction $(125 \mathrm{mg} / \mathrm{kg}, 250 \mathrm{mg} / \mathrm{kg}$ and $500 \mathrm{mg} / \mathrm{kg} \mathrm{b.w}$ ) in AI of both CETV and EETV treated groups. The changes in AI both in all the groups were expressed in Figure 2.

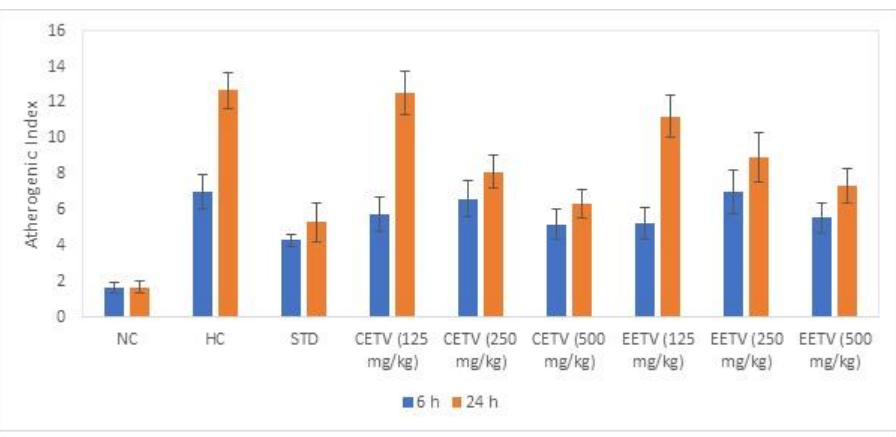

Figure 2 Effect of chloroform and ethanolic extract of $T$. villosa on Atherogenic index (AI) in hyperlipidemic rats of acute model.

\section{Chronic hyperlipidemia model}

In this model, all rats from group II to IX were fed with diet rich in cholesterol for 30 days. The serum lipid parameters analysed in all group of rats on day 0,15 and 30 were showed in Table 3 and Figure 3 . The results show that, on $15^{\text {th }}$ day of serum analysis there is a highly significant $(\mathrm{p}<0.001)$ rise in TC, VLDL-C, LDL-C TG levels and alleviation in HDL-C levels, in all the groups compared to $\mathrm{NC}$ group. After treating the rats with CETV and EETV at 3 different doses $(125$ $\mathrm{mg} / \mathrm{kg}, 250 \mathrm{mg} / \mathrm{kg}$ and $500 \mathrm{mg} / \mathrm{kg}$ b.w) exhibited remarkable effect on lipid parameters in dose dependent manner. On $30^{\text {th }}$ day, treatment groups at the dose of $250 \mathrm{mg} / \mathrm{kg}$ and $500 \mathrm{mg} / \mathrm{kg}$ b.w of both extracts exhibited significant decrease $(\mathrm{p}<0.001)$ in the serum TG, TC, LDL-C and VLDL-C along with slightly elevation ( $p<0.01$ in CETV and $p<0.05$ in EETV) in HDL levels. The results obtained where compared with Simvastatin (Standard) which showed significant modifications $(p<0.001)$ in the serum lipid parameters in treated group (STD).

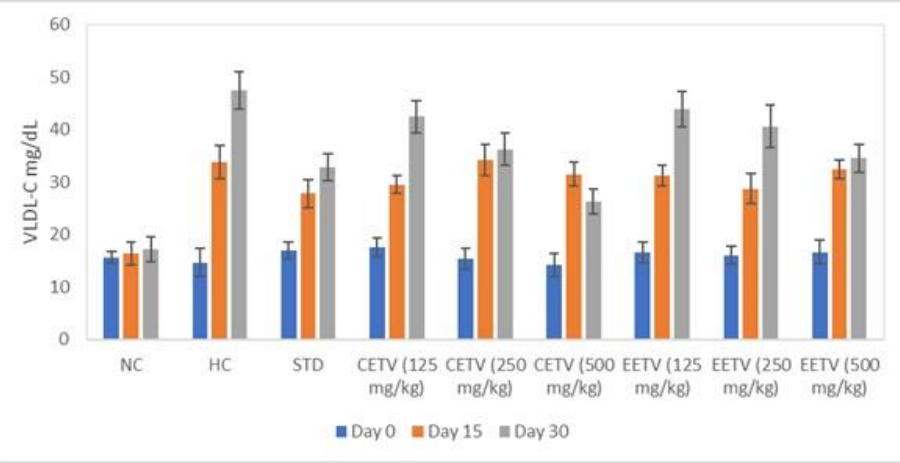

Figure 3 Effect of chloroform and ethanolic extract of $T$. villosa on VLDL-C level in hyperlipidemic rats of chronic model.

Similar to Triton model, Atherogenic index (AI) value were significantly increased in $\mathrm{HC}$ group in contrast to $\mathrm{NC}$ group. The group which received the standard drug observed highly significant dip in AI value and the treatment group (Group IV to IX) which received both CETV and EETV extracts $(125 \mathrm{mg} / \mathrm{kg}$, $250 \mathrm{mg} / \mathrm{kg}$ and $500 \mathrm{mg} / \mathrm{kg}$ ) respectively exhibited dose dependent decrease in the atherogenic index, is depicted in Figure 4.

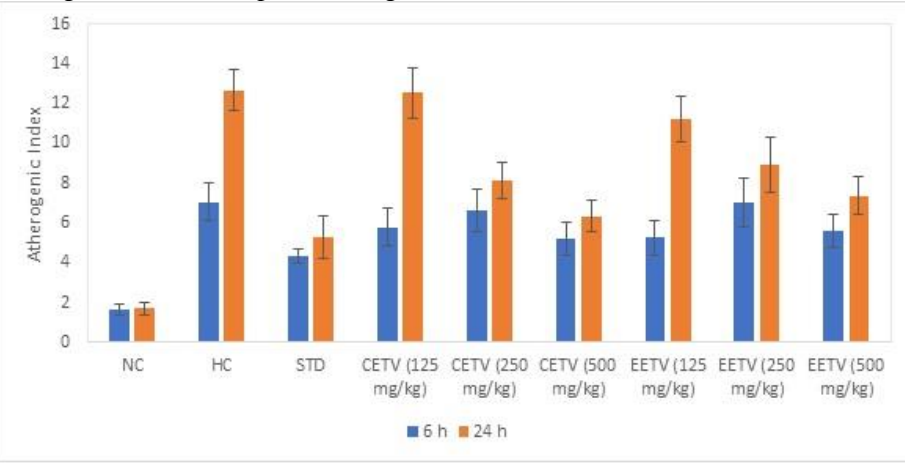

Figure 4 Effect of chloroform and ethanolic extract of T. villosa on Atherogenic index (AI) in hyperlipidemic rats of chronic model. 
Table 3 Effect of chloroform and ethanolic extract of T. villosa on lipid profile in hyperlipidemic rats of chronic model

\begin{tabular}{|c|c|c|c|c|c|c|c|c|c|c|c|c|c|c|c|c|c|c|c|c|c|c|c|c|}
\hline \multirow{3}{*}{$\begin{array}{l}\text { Groups } \\
\text { NC }\end{array}$} & \multicolumn{6}{|c|}{ TG mg/dL } & \multicolumn{6}{|c|}{ TC mg/dL } & \multicolumn{6}{|c|}{ LDL $\mathrm{mg} / \mathrm{dL}$} & \multicolumn{6}{|c|}{ HDL mg/dL } \\
\hline & \multicolumn{2}{|l|}{ Day 0} & \multicolumn{2}{|l|}{ Day 15} & \multicolumn{2}{|l|}{ Day 30} & \multicolumn{2}{|l|}{ Day 0} & \multicolumn{2}{|l|}{ Day 15} & \multicolumn{2}{|l|}{ Day 30} & \multicolumn{2}{|l|}{ Day 0} & \multicolumn{2}{|l|}{ Day 15} & \multicolumn{2}{|l|}{ Day 30} & \multicolumn{2}{|c|}{ Day 0} & \multicolumn{2}{|l|}{ Day 15} & \multicolumn{2}{|l|}{ Day 30} \\
\hline & $\begin{array}{l}78.21 \\
2.87\end{array}$ & \pm & $\begin{array}{l}82.18 \\
3.14\end{array}$ & \pm & $85.51 \pm 4$ & & $\begin{array}{l}95.25 \\
2.96\end{array}$ & \pm & $\begin{array}{l}97.15 \\
3.09\end{array}$ & \pm & $92.48 \pm 3$ & & $\begin{array}{l}44.13 \\
2.24\end{array}$ & \pm & $38.1 \pm 1$ & & $47.34 \pm 3$ & & $\begin{array}{l}40.46 \\
\pm 2.43\end{array}$ & & $\begin{array}{l}42.46 \\
2.09\end{array}$ & \pm & $\begin{array}{l}41.29 \\
3.27\end{array}$ & \pm \\
\hline $\mathrm{HC}$ & $\begin{array}{l}72.65 \\
3.54\end{array}$ & \pm & $\begin{array}{l}164.35 \\
3.62^{* * *(a)}\end{array}$ & \pm & $\begin{array}{l}236.13 \\
6.42^{* * *(a)}\end{array}$ & \pm & $\begin{array}{l}86.31 \\
3.67^{\text {ns }}\end{array}$ & \pm & $\begin{array}{l}151.21 \\
3.89^{* * *(a)}\end{array}$ & \pm & $\begin{array}{l}210.28 \\
5.27^{* * *(a)}\end{array}$ & \pm & $\begin{array}{l}40.78 \\
2.23\end{array}$ & \pm & $\begin{array}{l}79.23 \\
2.45^{* * *(a)}\end{array}$ & \pm & $\begin{array}{l}139.21 \\
4.35^{* * *(a)}\end{array}$ & \pm & $\begin{array}{l}38.12 \\
\pm 2.86\end{array}$ & & $\begin{array}{l}28.91 \\
2.98^{* * *(a)}\end{array}$ & \pm & $\begin{array}{l}22.95 \\
3.23^{* * *(a)}\end{array}$ & \pm \\
\hline STD & $\begin{array}{l}84.86 \\
3.11\end{array}$ & \pm & $\begin{array}{l}139.34 \\
3.21^{* * *(a)}\end{array}$ & \pm & $\begin{array}{l}163.91 \\
5.61^{* * *(b)}\end{array}$ & \pm & $\begin{array}{l}92.98 \\
2.56^{\text {ns }}\end{array}$ & \pm & $\begin{array}{l}147.67 \\
4.15^{* * *(a)}\end{array}$ & \pm & $\begin{array}{l}101.74 \\
3.04^{* * *(b)}\end{array}$ & \pm & $\begin{array}{l}36.16 \\
2.46\end{array}$ & \pm & $\begin{array}{l}76.09 \\
2.34^{* * *(a)}\end{array}$ & \pm & $\begin{array}{l}68.46 \\
3.16^{* * *(b)}\end{array}$ & \pm & $\begin{array}{l}41.35 \\
\pm 1.75\end{array}$ & & $\begin{array}{l}37.32 \\
1.21^{\text {ns }}\end{array}$ & \pm & $\begin{array}{l}43.41 \\
2.96^{* * * *(b)}\end{array}$ & \pm \\
\hline $\begin{array}{l}\text { CETV } \\
(125 \\
\mathrm{mg} / \mathrm{kg})\end{array}$ & $\begin{array}{l}87.68 \\
2.34\end{array}$ & \pm & $\begin{array}{l}149.41 \\
2.19^{* * *(a)}\end{array}$ & \pm & $\begin{array}{l}212.22 \\
7.51^{1 \mathrm{ss}}\end{array}$ & \pm & $\begin{array}{l}79.81 \\
2.29^{\text {ns }}\end{array}$ & \pm & $\begin{array}{l}155.65 \\
3.98^{* * *(a)}\end{array}$ & \pm & $\begin{array}{l}182.58 \\
4.12^{* *(b)}\end{array}$ & \pm & $\begin{array}{l}35.81 \\
2.08\end{array}$ & \pm & $\begin{array}{l}84.73 \\
3.67^{* * *(a)}\end{array}$ & \pm & $\begin{array}{l}126.51 \\
4.25^{\mathrm{ns}}\end{array}$ & \pm & $\begin{array}{l}35.19 \\
\pm 2.98\end{array}$ & & $\begin{array}{l}29.12 \\
2.23^{* * *(a)}\end{array}$ & \pm & $\begin{array}{l}28.76 \\
3.08^{\text {ns }}\end{array}$ & \pm \\
\hline $\begin{array}{l}\text { CETV } \\
(250 \\
\mathrm{mg} / \mathrm{kg})\end{array}$ & $\begin{array}{l}76.52 \\
3.19^{\text {ns }}\end{array}$ & \pm & $\begin{array}{l}174.34 \\
4.61^{* *(a)}\end{array}$ & \pm & $\begin{array}{l}180.06 \\
6.25^{* * *(b)}\end{array}$ & \pm & $\begin{array}{l}89.48 \\
2.36^{\text {ns }}\end{array}$ & \pm & $\begin{array}{l}149.23 \\
2.43^{* *(a)}\end{array}$ & \pm & $\begin{array}{l}156.35 \\
3.58^{* * *(b)}\end{array}$ & \pm & $\begin{array}{l}38.18 \\
3.24\end{array}$ & \pm & $\begin{array}{l}79.92 \\
3.26^{* * *(a)}\end{array}$ & \pm & $\begin{array}{l}110.71 \\
4.64^{* * *(b)}\end{array}$ & \pm & $\begin{array}{l}42.46 \\
2.32\end{array}$ & \pm & $\begin{array}{l}36.75 \\
1.30^{*(a)}\end{array}$ & \pm & $\begin{array}{l}38.54 \\
2.89^{* *(b)}\end{array}$ & \pm \\
\hline $\begin{array}{l}\text { CETV } \\
(500 \\
\mathrm{mg} / \mathrm{kg})\end{array}$ & $\begin{array}{l}72.86 \\
2.24^{\mathrm{ns}}\end{array}$ & \pm & $\begin{array}{l}159.01 \\
3.69^{* * *(a)}\end{array}$ & \pm & $\begin{array}{l}132.25 \\
4.68^{* * *(b)}\end{array}$ & \pm & $\begin{array}{l}84.21 \\
1.91^{\text {ns }}\end{array}$ & \pm & $\begin{array}{l}160.31 \\
3.14^{* * *(a)}\end{array}$ & \pm & $\begin{array}{l}137.52 \\
2.96^{* * *(b)}\end{array}$ & \pm & $\begin{array}{l}42.12 \\
1.84\end{array}$ & \pm & $\begin{array}{l}82.12 \\
4.34^{* * *(a)}\end{array}$ & \pm & $\begin{array}{l}89.69 \\
3.51^{* * *(b)}\end{array}$ & \pm & $\begin{array}{l}44.54 \\
\pm 3.11\end{array}$ & & $\begin{array}{l}31.45 \\
1.55^{* * *(a)}\end{array}$ & \pm & $\begin{array}{l}40.25 \\
3.13^{* *(b)}\end{array}$ & \pm \\
\hline $\begin{array}{l}\text { EETV } \\
(125 \\
\mathrm{mg} / \mathrm{kg})\end{array}$ & $\begin{array}{l}82.82 \\
3.14^{\text {ns }}\end{array}$ & \pm & $\begin{array}{l}156.32 \\
3.45^{* *(a)}\end{array}$ & \pm & $\begin{array}{l}219.46 \\
5.28^{\mathrm{ns}}\end{array}$ & \pm & $\begin{array}{l}94.28 \\
3.14^{\mathrm{ns}}\end{array}$ & \pm & $\begin{array}{l}140.83 \\
2.34^{* * *(a)}\end{array}$ & \pm & $\begin{array}{l}190.84 \\
4.12^{\mathrm{ns}}\end{array}$ & \pm & $\begin{array}{l}45.88 \\
3.12\end{array}$ & \pm & $\begin{array}{l}90.08 \\
3.32^{* * *(a)}\end{array}$ & \pm & $\begin{array}{l}130.56 \\
4.12^{\mathrm{ns}}\end{array}$ & \pm & $\begin{array}{l}39.55 \\
1.30\end{array}$ & \pm & $\begin{array}{l}25.19 \\
2.37^{* * *(a)}\end{array}$ & \pm & $\begin{array}{l}32.26 \\
2.79^{\mathrm{ns}}\end{array}$ & \pm \\
\hline $\begin{array}{l}\text { EETV } \\
(250 \\
\mathrm{mg} / \mathrm{kg})\end{array}$ & $\begin{array}{l}80.63 \\
3.28^{\text {ns }}\end{array}$ & \pm & $\begin{array}{l}145.18 \\
3.18^{* * *(a)}\end{array}$ & \pm & $\begin{array}{l}203.21 \\
6.41^{* *(b)}\end{array}$ & \pm & $\begin{array}{l}95.21 \\
3.71^{\text {ns }}\end{array}$ & \pm & $\begin{array}{l}165.28 \\
4.04^{* * *(a)}\end{array}$ & \pm & $\begin{array}{l}182.64 \\
4.90^{* *(b)}\end{array}$ & \pm & $\begin{array}{l}39.16 \\
2.28\end{array}$ & \pm & $\begin{array}{l}87.86 \\
3.14^{* * *(a)}\end{array}$ & \pm & $\begin{array}{l}122.41 \\
3.95^{*} \text { (b) }\end{array}$ & \pm & $\begin{array}{l}43.06 \\
\pm 2.33\end{array}$ & & $\begin{array}{l}33.64 \\
1.19^{* *(a)}\end{array}$ & \pm & $\begin{array}{l}35.41 \\
2.43^{*(b)}\end{array}$ & \pm \\
\hline $\begin{array}{l}\text { EETV } \\
(500 \\
\mathrm{mg} / \mathrm{kg})\end{array}$ & $\begin{array}{l}83.14 \\
2.68\end{array}$ & & $\begin{array}{l}162.54 \\
2.76^{* *(a)}\end{array}$ & \pm & $\begin{array}{l}170.55 \\
4.97^{* * *(b)}\end{array}$ & \pm & $\begin{array}{l}90.29 \\
2.82^{\text {ns }}\end{array}$ & \pm & $\begin{array}{l}159.48 \\
2.34^{* * *(a)}\end{array}$ & \pm & $\begin{array}{l}165.38 \\
5.74^{* * *(\mathrm{~b})}\end{array}$ & \pm & $\begin{array}{l}38.85 \\
2.24^{\text {ns }}\end{array}$ & \pm & $\begin{array}{l}72.92 \\
2.87^{* * *(a)}\end{array}$ & \pm & $\begin{array}{l}106.54 \\
3.23^{* * *(b)}\end{array}$ & \pm & $\begin{array}{l}37.46 \\
\pm 1.48\end{array}$ & & $\begin{array}{l}25.28 \\
1.64^{* * *(a)}\end{array}$ & \pm & $\begin{array}{l}34.32 \\
2.05^{*(b)}\end{array}$ & \pm \\
\hline
\end{tabular}

Values are expressed as Mean \pm SEM;

${ }^{a}$ Values were against normal control (NC).

${ }^{\mathrm{b}}$ Values were against hyperlipidemic control (HC)

Values are statistically significant at $* * * \mathrm{p}<0.001,{ }^{*} \mathrm{p}<0.01 * \mathrm{p}<0.05$ and ${ }^{\mathrm{ns}}$ nonsignificant

NC: Normal control, HC: Hyperlipidemic control, STD: Standard control (Simvastatin), CETV: Chloroform extract of T.villosa, EETV: Ethanolic extract of T.villosa, TC: Total cholesterol, TG: Triglycerides, HDL-C: High density lipoprotein cholesterol, LDL-C: Low density lipoprotein cholesterol

\section{DISCUSSION}

Hyperlipidemia is termed as disorder of lipid metabolism characterised by abnormal rise of serum lipid parameter such as TG, TC, LDL-C and VLDL-C, which are accompanied by atherosclerosis and many other CVD (Sikarwar $\boldsymbol{e} t$ al., 2014; Khazaal, 2013). Increase in the mortality due to vascular disease and atherosclerosis is a major concern. Thus, prevention and control of hyperlipidemia is prerequisite to reduce complications (Touiss et al., 2019). However, there are many treatment options readily available to treat hyperlipidemia but herbal medicines are extensively used, due to their lesser side effects.

The present study was aimed to demonstrate the antihyperlipidemic effect of Tephrosia villosa using triton WR-1339 and high cholesterol diet model. Triton WR-1339, an anionic surfactant increases TC, VLDL-C, LDL-C and TG levels. The underlying mechanism would be by inhibiting lipoprotein lipase enzyme (LPL) and by blocking the triacyl glycerol rich lipoproteins from peripheral tissues (Gupta et al., 2018). Inhibition of LPL enzyme leads to hydrolysis of TGs present in plasma as in the form of chylomicron and VLDL-C lipoproteins. On top of it, 3-hydroxy-3-methyl-glutaryl CoA reductase (HMG-CoA reductase) and hepatic synthesis of cholesterol were induced by triton (E Souza et al., 2017). In this study, it was observed that there was significant increase in TC, VLDL-C, LDL-C and TG and declined HDL-C levels after triton WR-1339 administration, whereas CETV and EETV treated rats reversed the triton effect significantly when compared to hyperlipidemic control (HC) rats.

Administrations of high fat-diet (HFD)/cholesterol diet causes increase in all serum lipid parameters in rats similar to the diseased condition like in humans. Thus, it is one of the models used to study the hypolipidemic effect of the plant. Upon HFD in rats, it increases the availability of acetyl coenzyme A, a prime substrate for synthesis of cholesterol by the oxidation of fatty acid (Irudayaraj $\boldsymbol{e}$ al., 2013). It is also believed that it modifies the circulation of arginine rich apoprotein which is usually responsible for migration of all lipoproteins in plasma (Murugaiyah et al., 2018). The above proposed mechanism explains the induction of hyperlipidemia in rats. In our study both extracts were able to deplete the TC, VLDL-C, LDL-C and TG levels, critically when hypercholesteremia was induced by cholesterol diet.

The decline of serum cholesterol was accompanied with reduction in the LDL-C fraction. Through hepatic LDL receptors, LDL-C undergo catabolism leading to formation of bile salts and secreted into the bile. HDL-C is a "cardio protective lipid" and is believed that increase in HDL-C levels reduced the risk of atherosclerosis through reverse cholesterol transport (RCT). Many studies suggested that that HDL-C mediated RCT occurs by three process: it translocates the cholesterol from peripheral cell to circulating lipoprotein in to the liver for catabolism and excretion, mobilization of cellular cholesterol results in formation of HDL-C particles and to the liver it transports the HDL-C derived cholesterol (Millar et al., 2017). Presence of flavonoids and polyphenols in Tephrosia villosa leads to increase the HDL-C levels due to potentiation of lecithin acyl transferase (LCAT) activity and constraining the HMG CoA reductase activity. LCAT plays a crucial part in the incorporation of free cholesterol into HDL-C (this may elevate HDL-C) and moving it back later in liver cells, while inhibition of HMG-CoA reductase hinders the cholesterol synthesis (Zeka et al., 2017). The above two mechanism plays a synergistic activity in increasing the HDL-C level, lowering of which is associated with ischemic heart disease.

In addition to the lowering of serum lipid profile, CETV and EETV showed effect on atherogenic index (AI). AI is complex made up of TG and HDL-C gives correlation between atherogenic and protective lipids and a strong predictor of the $\mathrm{CAD}$ and atherosclerosis. Reductions in the $\mathrm{AI}$ is a positive physiological effect because it is believed that high plasma HDL-C levels and low LDL-C levels is desired to prevent the atherogenesis (Oršolić et al., 2019; Niroumand $\boldsymbol{e t}$ al., 2015). During the study both extracts showed significant decrease in the Al thus maintained the LDL-C/HDL-C ratio which explains the action of lipid lowering activity of extracts.

In the present study both extracts CETV and EETV elucidated the antihyperlipidemic activity by reducing the serum cholesterol. It significantly suppressed TG, TC, LDL-C and VLDL-C levels and induce high serum concentration of HDL-C. Thus, suggest the partial restoration of catabolism of TGs, underlying mechanism is not explained by this study. The preliminary phytochemical study report provides the additional support for Tephrosia villosa being used traditionally for treating hyperlipidemia. Earlier studies on Tephrosia villosa established the presence of various flavonoids, triterpenoid (Lupenone), triterpene (Lupeol) and sterol (Stigmasterol). Hence, the above mentioned phytoconstituents were proven for its anti-hypercholesterolemic property by earlier studies (Sudhahar et al., 2006; Chandler et al., 1979; Madhusudhana et al., 2010). Nevertheless, hypothesized by other studies that it may be due to stimulation of lipoprotein lipase (LPL) and presence of phenolic compounds (Gopichandchinta et al.,2009).

Based on various studies, the extraction of polyphenols and supplementary phytoconstituents will be influenced by their diverse structures especially in phenolic compounds. The Polyphenols have better solubility in more polar solvents in nature (Aires et al., 2017). Hence, in this study both the extracts showed good antihyperlipidemic activity but chloroform extract showed slightly better than ethanolic extract.

\section{Study limitation}

The future perspective of the study would be isolation and characterisation of the compounds from Tephrosia villosa extracts to explain the mechanism of hypolipidemic activity and using the extracts of different parts of plants can be done to isolate more precise compounds for their potent activity.

\section{CONCLUSION}

In conclusion to the results obtained both chloroform and ethanolic extract of Tephrosia villosa was able to mitigate the levels of TG, TC, VLDC-C and LDC$\mathrm{C}$, and contribute to rise the HDL-C levels in Triton WR-1339 induced and Cholesterol diet induced hyperlipidemic rats. This effect may be due to their influence in some mechanisms like inhibition of HMG CoA reductase, activation of lipoprotein lipase and LCAT. Thus, it can be considered as antihyperlipidemic agent in the treating hyperlipidemia. 
Acknowledgement: The authors urge to convey their gratitude to the Management of Dayananda Sagar University, Bengaluru for providing facilities and necessary support for pursuing the research work.

\section{REFERENCES}

Aires, A. (2017). Phenolics in foods: Extraction, analysis and measurements. Phenolic Compounds, 61-88. https://doi.org/10.5772/66889 Chandler, R. F., Hooper, S. N., \& Ismail, H. A. (1979). Antihypercholesterolemic studies with sterols: $\beta$-sitosterol and stigmasterol. Journal of pharmaceutical sciences, 68(2), 245-247.

Dubey, A. K., Devi, A., Kutty, G., \& Shankar, R. P. (2005). Hypolipidemic activity of Ginkgo biloba extract, EGb 761 in hypercholesterolemic wistar rats. IJPT, 4(1), 9-12.

E Souza, B. S. F., Carvalho, H. O., Ferreira, I. M., da Cunha, E. L., Barros, A. S., Taglialegna, T., \& Carvalho, J. C. T. (2017). Effect of the treatment with Euterpe oleracea Mart. oil in rats with Triton-induced dyslipidemia. Biomedicine \& Pharmacotherapy, 90, 542-547. https://doi:10.1016/j.biopha.2017.04.005

Gokhale, S.B., Gokhale, A., Kulkarni, Y., Yele, S., (2016). Experimental Pharmacognosy, Second ed., Pune, Nirali Prakashan.

Gopichandchinta, Prashanthi, K., Pushpa Kumari, B., Sujata, D., Ranganayakulu, D., \& Venkateswarlu, M. (2009). Hypolipidemic activity of the aqueous extract from the Morinda citrifoloa leaves in triton induced hyperlipidemic rats. Pharmacologyonline, 3, 9-28.

Guideline, O. E. C. D. (2002). Test No. 423: Acute Oral Toxicity-Acute Toxic Class Method.

Guptha, B. H. M. R. K., Kadali, S. R. M., Vijay, K. M., \& Revanasiddappa, B. C. (2018). Antihyperlipidemic activity of chloroxylonswietenia in triton WR1339 induced hyperlipidemia. International Journal of Basic \& Clinica Pharmacology, 7(3), 518-523. $\quad$ https://dx.doi.org/10.18203/2319 2003.ijbcp20180667

Hmidani, A., Bourkhis, B., Khouya, T., Harnafi, H., Filali-Zegzouti, Y., \& Alem, C. (2020). Effect of Phoenix dactylifera seeds (dates) extract in triton WR-1339 and high fat diet induced hyperlipidaemia in rats: A comparison with simvastatin. Journal of $\quad$ Ethnopharmacology, 112961. https://doi.org/10.1016/j.jep.2020.112961

Ibrahim, A. Y., Hendawy, S. F., Elsayed, A. A., \& Omer, E. A. (2016) Evaluation of hypolipidemic Marrubium vulgare effect in Triton WR-1339 induced hyperlipidemia in mice. Asian Pacific journal of tropical medicine, 9(5), 453-459. https://doi.org/10.1016/j.apjtm.2016.03.038

Irudayaraj, S. S., Sunil, C., Duraipandiyan, V., \& Ignacimuthu, S. (2013). In vitro antioxidant and antihyperlipidemic activities of Toddalia asiatica (L) Lam. leaves in Triton WR-1339 and high fat diet induced hyperlipidemic rats. Food and chemical toxicology, 60, 135-140. https://doi.org/10.1016/j.fct.2013.07.035

Karr, S. (2017). Epidemiology and management of hyperlipidemia. The American journal of managed care, 23(9 Suppl), S139.

Khazaal, M. S. (2013). Atherogenic Index of Plasma (AIP) as a parameter in predicting cardiovascular risk in males compared to the conventional dyslipidemic indices (cholesterol ratios). Karbala journal of medicine, 6(1), 1506 1513.

Kreisberg, R. A., \& Reusch, J. E. B. (2005). Hyperlipidemia (High Blood Fat) The Journal of Clinical Endocrinology \& Metabolism, 90(3), 0-0. https://doi:10.1210/jcem.90.3.9991

Krishnasamy, R., \& Periyasamy, S. (2019). Regulating role of ethyl acetate fraction of Tephrosia tinctoria pers. in carbohydrate metabolism and oxidative stress in diabetic rats. Biomedicine \& Pharmacotherapy, 114, 108842

Kumar, G. V. (2016). Antihyperlipidemic Activity of Leaf Extracts of Leucas aspera Linn. against Dexamethasone-induced Hyperlipidemia in Rats. Asian Journal of Pharmaceutics, 10(03).

Madhusudhana, J., Reddy, R. N., Reddy, B. A. K., Reddy, M. V. B., Gunasekar, D., Deville, A., \& Bodo, B. (2010). Two new geranyl flavanones from Tephrosia villosa. Natural product research, 24(8), 743-749.

Mani, A. S., Prasad, Y. R., Siddhanadham, A. S., \& Aparna, B. (2017) Phytochemical and anti-oxidant activity screening of chloroform leaf and aerial part extracts of Tephrosia villosa. World J. of Pharmaceutical, 13(9), 181-184.

Millar, C. L., Duclos, Q., \& Blesso, C. N. (2017). Effects of dietary flavonoids on reverse cholesterol transport, HDL metabolism, and HDL function. Advances in Nutrition, 8(2), 226-239. https://doi.org/10.3945/an.116.014050

Murugaiyah, V., Saeed, M. A. A., Kuong, Y. M., Murugesu, K., Parasuraman, S., Asmawi, M. Z., \& Sadikun, A. (2018). Lipid-lowering effect of hydroalcoholic extracts of Gynura procumbens in Chemical-and High-fat diet-induced $\begin{array}{lll}\text { hyperlipidemic } & \text { rats. Pharmacognosy } & \text { Magazine, 14(55), }\end{array}$ https://doi.org/0.4103/pm.pm_451_17

Nelson, R. H. (2013). Hyperlipidemia as a Risk Factor for Cardiovascular Disease. Primary Care: Clinics in Office Practice, 40(1), 195-211. https://doi:10.1016/j.pop.2012.11.003

Niroumand, S., Khajedaluee, M., Khadem-Rezaiyan, M., Abrishami, M., Juya, M., Khodaee, G., \& Dadgarmoghaddam, M. (2015). Atherogenic Index of Plasma (AIP): A marker of cardiovascular disease. Medical journal of the Islamic Republic of Iran, 29, 240.
Oršolić, N., Landeka Jurčević, I., Đikić, D., Rogić, D., Odeh, D., Balta, V., ... \& Jutrić, D. (2019). Effect of propolis on diet-induced hyperlipidemia and atherogenic indices in mice. Antioxidants, 8(6), 156 https://doi.org/10.3390/antiox8060156

Sampathkumar, M. T., Kasetti, R. B., Nabi, S. A., Sudarshan, P. R., Swapna, S., \& Apparao, C. (2011). Antihyperlipidemic and antiatherogenic activities of Terminalia pallida Linn. fruits in high fat diet-induced hyperlipidemic rats. Journal of Pharmacy and Bioallied Sciences, 3(3), 449. https://doi.org/10.4103/0975-7406.84464

Samuel, V. J., Mahesh, A. R., \& Murugan, V. (2019). Phytochemical and pharmacological aspects of Tephrosia genus: A brief review. J Appl Pharm Sci, 9(03), 117-125. https://doi.org/10.7324/JAPS.2019.90317

Santini, A., \& Novellino, E. (2016). Nutraceuticals in hypercholesterolaemia: an overview. British Journal of Pharmacology, 174(11), 1450-1463. https://doi.org/10.1111/bph.13636

Sikarwar, M. S., \& PATIL, M. B. (2014). Antihyperlipidemic Activity of Pongamia pinnata Leaf Extracts. Turk J Pharm Sci, 11(3), 329-338.

Sudhahar, V., Kumar, S. A., \& Varalakshmi, P. (2006). Role of lupeol and lupeol linoleate on lipemic-oxidative stress in experimental hypercholesterolemia. Life sciences, 78(12), 1329-1335. https://doi.org/10.1016/i.lfs.2005.07.011

Touiss, I., Harnafi, M., Khatib, S., Bekkouch, O., Ouguerram, K., Amrani, S., \& Harnafi, H. (2019). Rosmarinic acid-rich extract from Ocimum basilicum L. decreases hyperlipidemia in high fat diet-induced hyperlipidemic mice and prevents plasma lipid oxidation. Physiology and Pharmacology, 0-0.

Vijaya, C., Ramanathan, M., \& Suresh, B. (2009). Lipid lowering activity of ethanolic extract of leaves of Aegle marmelos (Linn.) in hyperlipidaemic models of Wistar albino rats. Indian journal of experimental biology, 47(3), 182-185.

Zeka, K., Ruparelia, K., Arroo, R. R., Budriesi, R., \& Micucci, M. (2017). Flavonoids and their metabolites: prevention in cardiovascular diseases and diabetes. Diseases, 5(3), 19. https://doi.org/10.3390/diseases5030019

Zodda, D., Giammona, R., \& Schifilliti, S. (2018). Treatment Strategy for Dyslipidemia in Cardiovascular Disease Prevention: Focus on Old and New Drugs. Pharmacy, 6(1), 10. https://doi.org/10.3390/pharmacy6010010 